\title{
Digital Analysis of Changes in Chronic Wounds through Image Processing
}

\author{
K Sundeep Kumar ${ }^{1}$ and B. Eswara Reddy ${ }^{2}$ \\ ${ }^{1}$ Associate Professor, Dept. of CSE, CMR Institute of Technology, Bangalore, India \\ ${ }^{2}$ Professor, Dept. of CSE, JNTU College of Engineering Anantapur, 515002, India \\ ${ }^{1}$ sundeepkk@yahoo.co.in, ${ }^{2}$ eswarcsejntu@gmail.com
}

\begin{abstract}
Measurement of wound healing status is very important for monitoring and evaluation of progress in an individual patient. Thus wound classification is a vital step in the development of an automatic measurement system for wound healing assessment. In the existing system a RGB (Red, Green and Blue) histogram distributions of pixel values from wound colour images is being used in the new tissue classification protocol [1],[4]. This protocol has been carried out using the KNN classifier and results show that the in the proposed system the existing system protocol is integrated with another protocol i.e., fuzzy classification for maximum probability. Thus the maximum probable result is considered as final classification of the resultant wound type depending whether the wound categorizes under the acute or the chronic wound and denotes the healing period.
\end{abstract}

Keywords: Image processing, tissue classification, leg ulcers, chronic wounds, fuzzification wound healing, wound assessment for chronic wounds (WAC)

\section{Introduction}

Wound healing is a complex and dynamic process, which varies with the changing health status of the individual. The knowledge about the healing stages i.e. haemostasis, inflammation; granulation and maturation are the phases of wound healing provide a framework for an understanding of the basic principles of wound healing and the phases of healing. This helps the health care professionals to understand and to develop a skill which is required to take care about the wound, body and to overcome with the task of complex tissue repair. 


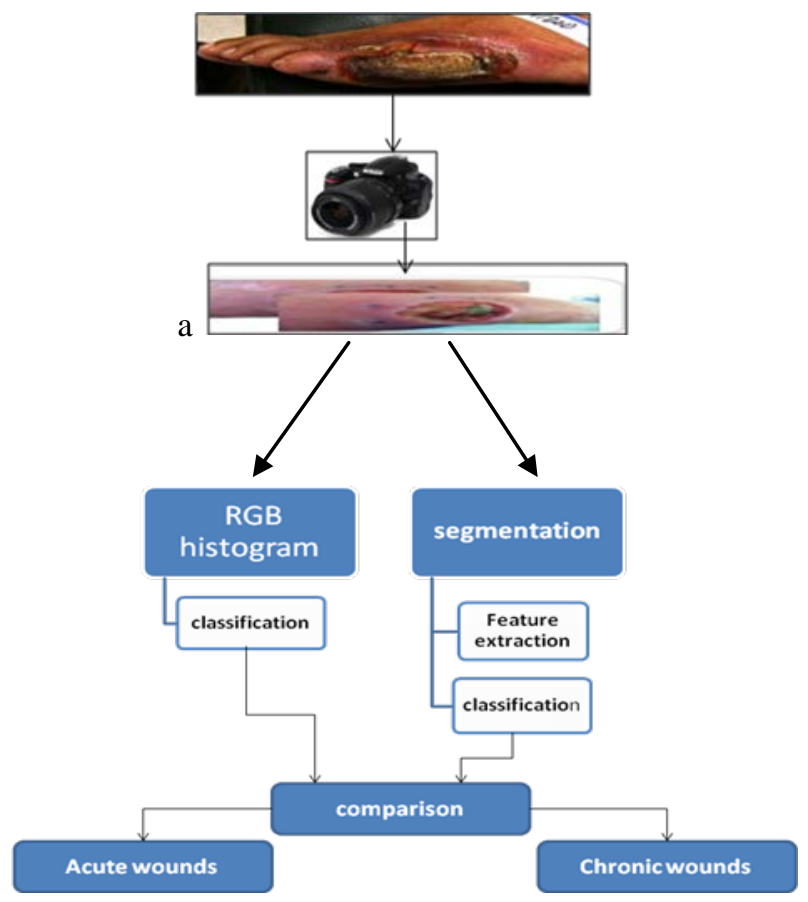

Figure 1. Assessment of Wounds

Wound image analysis finds its application in healthcare to monitor the extent of wound healing and to decide the course of treatment. To demonstrate the effects of a new drug, in wound healing techniques, the wound surface area is one of the most important variables. The healing process is a phenomenon which can be visually assessed by an observer but in qualitative terms only. Manual wound measurement is done by tracing the wound boundary and then calculating the area of wound. Thus, are errors prone and is a tedious task. Therefore we use this concept wound assessment for chronic wounds. In the Figure 1 an image database is created acquiring images of various wounds of various patients, certain pre-processing techniques and other cropping of image to select only the wound area to be focused on along with their respective RGB values. Thus by using K-NN classifier and Fuzzy classifier in this paper we would denote the type of wound. Since acute wounds are minor, easily heals, and does not come with any major stages of healing. In this paper, we focus mainly on chronic wounds that are mainly of the diabetic ulcers, venous ulcers and pressure ulcers (bedsores) and find the approximate percentage of healing.

Monitoring wound healing process is a tedious task for clinicians and nurses as it is necessary to periodically assess the wounds. All the types of wounds are concerned not only chronic wounds but also ulcers, cuts or burns, any trauma or surgical wounds, and dermatological lesions in tissues. Moreover, wound care is expensive: according to a report published by the NIGMS in 2008 in the USA, chronic wounds cost the nation \$20 billion to $\$ 25$ billion and acute or traumatic wounds add another $\$ 7-\$ 10$ billion to the bill annually, cause the healing process can last several months; with the ageing of the population this cost will necessarily increase by $25 \%$ over the next 10 years.

As health care cost needs to drastically reduce, there is a growing demand for patients to be given care at home; wound monitoring could be carried out from a distance, outside a environment of a hospital, in some private homes properly equipped for telemedicine practice required. Pioneer experiments in this area consisted simply in uploading images to a web site 
where a physician could view the data at his convenience. In more recent studies, image processing has been added but it provides only ulcer stage grading. The quantitative assessment of chronic wounds still relies on visual inspection and manual techniques to describe the shape of the wound (perimeter, surface, depth) and the biological nature of the skin tissues (percentage of each class, wound severity, burn degree).

Wound dimensions and shape are currently measured with an ordinary ruler, or sketches on cross-ruled sheets, serum injection or certain alginate mouldings [4]. Assessing certain type and the proportion of tissues likewise remains highly empirical as evaluation is performed visually and then recorded on a red-yellow-black scale corresponding respectively to the dominant colour of the different tissues found on a wound: granulation, slough and necrosis tissue. Healing is a complex cascade of cellular events operating to reconstruct damaged tissues, and is also an individual process that exhibits considerable inter-patient variability. As different tissues may overlap and it may be difficult to distinguish, wound assessment is not direct. The lack of necessary data affects coordination of care staff and hinders clinical studies focused on the healing. Digital cameras are used widely clinical centers, and only for basic patient data recording and not image processing, the wound therapeutic follow-up is mainly done by nurses.

Several studies have tackled the problem of wound assessment; certain attempts have failed to provide a robust tool for automatic tissue classification. Results remain very dependent on image capture conditions, several features contribute to make automatic classification tedious. First, wound image acquisition requires technical skill, especially in the patient rooms where lighting is not controlled. At a close range, the depth of field remains always limited in macro-mode, ambient light is not sufficient and may easily result in fuzzy images. Thus, a patient is rarely able to maintain a convenient posture for a snapshot. After the image is captured and image compression, is an important requirement for data management the wound area must be extracted from the image.

Early attempts to accomplish this by using colour measurements did not completely succeed, as reported by and have been replaced by semi-au-somatic methods by spline fitting from a set of clicked boundary points or snakes on a manually specified closed contour[5]. As all the photos are taken with the same camera and lighting during experimentation. Colour correction is mostly neglected, making it impossible to process images from other care centers correctly. Sometimes, a portion white patch is included in the field of view to estimate the white balance. More rarely a colour pattern is used for enhanced colour correction, gives access to the scale factor for dimensional measurements.

The colour analysis required for tissue classification over the wound is a difficult task. As any direct classification on the pixels with simple thresholds on separate colour components proved to be inefficient due to the variability and non homogeneity of tissues, spatial continuity has to be searched for through a segmentation process. At close range, the depth of field remains always limited in macro-mode, ambient light is not sufficient and may easily result in fuzzy images which have been captured accurately[6]. Colour histograms are typical of such statistical data gathering as tissue descriptors, but it has been proved that tissues could still not be classified robustly in a large collection of images. The best results are obtained when the classification was limited to two types of tissue or by multiplying the tissue classes using hybrid classes.

To improve the results, the texture parameters have finally been added several classification tools have also been addressed and supervised ones have been shown to surpass the others In many studies, assessment of the tissue type is not achieved (only the grade or the surface of the wound is provided) or only partially. 


\section{Wound Assessment}

In the existing system seen before, the type of wound considered for processing is leg ulcers [1], wherein the RGB Histogram of the given wound image is determined, based on which the KNN classifies the given data as to which stage of healing is the given wound in. Stages are namely - granulation and slough, granulation and necrotic, granulation and epithelial, slough and necrotic, slough and epithelial in Figure 2 below.

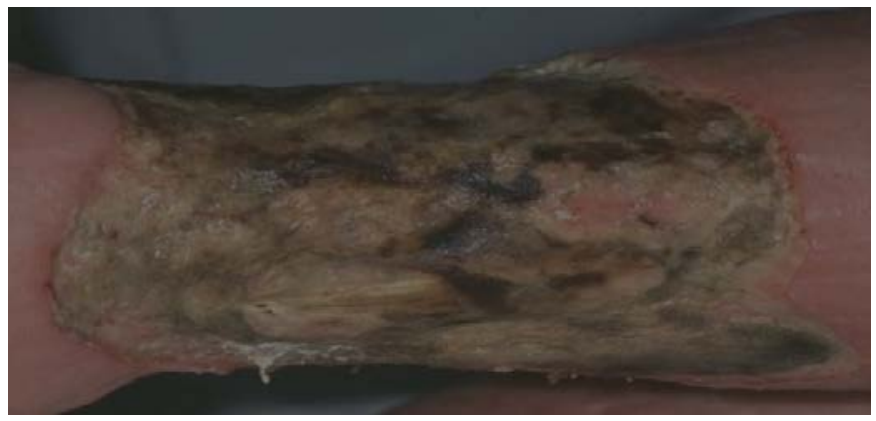

Figure 2. Stages of Healing Tissues of Leg Ulcer

In order to overcome the disadvantages of the existing system, the proposed system is modified by integrating the existing protocol with another protocol for accurate classification for a larger database of the wounds. In the protocol, the given wound image is segmented to identify the Region of Interest (ROI) to extract the features, which is then subjected to nearest neighbour for Classification.

In this methodology, integration of the two protocols will enables us to classify the type of given wound into chronic using assessment, and also in what stage of healing is the wound is in, hence the result will be more accurate and robust classification. The research of image processing laboratories involved in colour image processing applied to skin wounds, cuts lesions. The lack of non-invasive methods in the evaluation wound repair is a major obstacle in acquiring quantitative data in clinical trials. The role of colour image processing is the most acceptable thus image acquisition, digitisation and use of various image processing algorithms in the analysis of wounds and lesions [2].

Colour image processing has many advantages over human wound assessment for chronic wounds (WAC) and skin lesions digital image processing techniques are objective and producible again. Colour image processing has significant feature, since the analysis and comparison of colour images is a task which humans find particularly difficult. Feature extraction methodologies analyze objects and images to extract the most prominent features that are representative of the various classes of objects with the current technological trends in computer hardware and scanners, computerised systems are becoming easily affordable. Features are used as inputs to classifiers that assign them to the class that they represent. Thus the methodology as follows in Figure 3 which denotes the wound assessment for chronic wounds

\section{Methodology}

Module 1: Image Acquisition and Pre-Processing techniques - Wound database is created by acquiring images of various wounds of various patients. For the given wound image the pre-processing techniques are applied to remove the noise and hairs on the skin, so that the wound is distinct. 


\section{Protocol -1}

Module 2: RGB Histogram - Obtaining the different RGB values. These values are then used to identify different wounds. These values can then be used for training of the KNN and fuzzy values.

\section{Protocol - 2}

Module 3: Segmentation to ROI - Image segmentation is a process of extracting from the image domain one or more connected regions satisfying uniformity (homogeneity) criterion which is based on feature(s) derived from spectral components shown in Figure 8. These components are defined in a chosen colour space model. The segmentation process can be augmented by some additional knowledge about the objects in the scene such as geometric and optical properties.

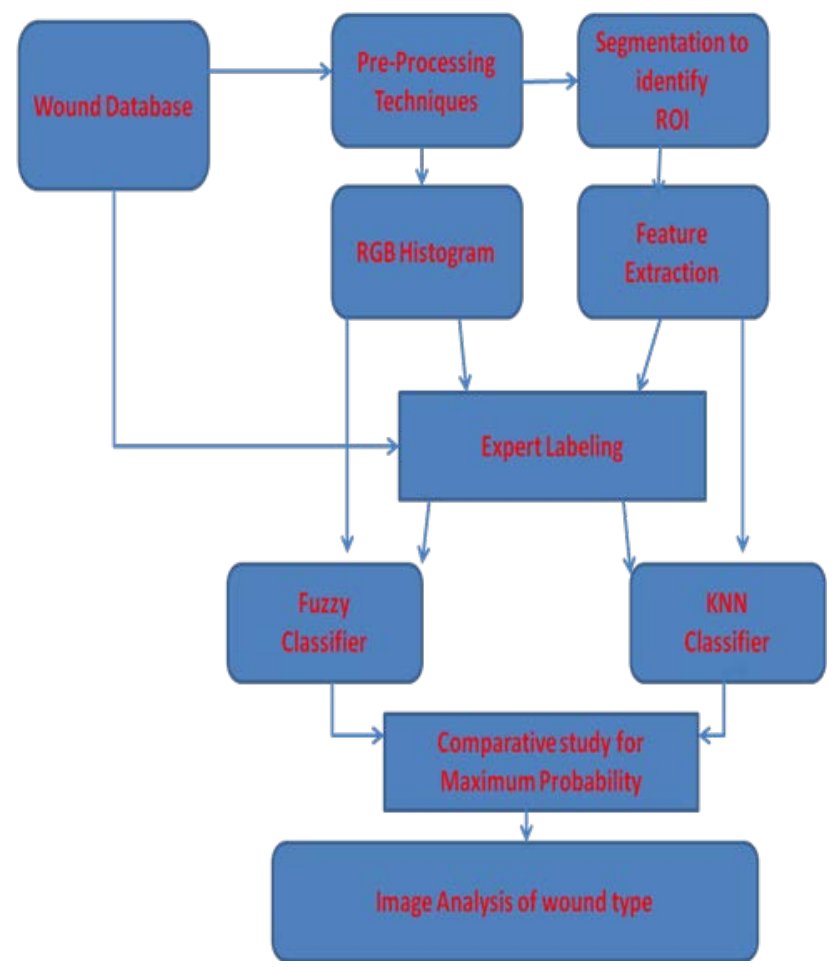

Figure 3. Modules of Wound Assessment for Chronic Wounds

Module 4: Feature Extraction - Features, characteristics of the objects of interest, if selected carefully represents the maximum relevant information that the image has to offer for a complete characterization a lesion. Feature extraction methodologies analyze objects and images to extract the most prominent features that are representative of the various classes of objects. Features are used as linguistic inputs to classifiers that assign them to the class that they represent. In this Work intensity histogram features and Gray Level Co-Occurrence Matrix (GLCM) features are extracted. The intensity histogram features like mean, variance, entropy etc. The Following GLCM features are considered: Autocorrelation, Correlation, Homogeneity etc. 
Module 5: K-NN Classifier - K-nearest neighbour algorithm is a method for classifying objects based on closest training examples in the feature space. K-nearest neighbour algorithm is among the simplest of all machine learning algorithms. Training process for this algorithm only consists of storing feature vectors and labels of the training images. In the classification process, the unlabelled query point is simply assigned to the label of its $\mathrm{k}$ nearest neighbours. Typically the object is classified based on the labels of its $\mathrm{k}$ nearest neighbours by majority vote. If $\mathrm{k}=1$, the object is simply classified as the class of the object nearest to it. When there are only two classes, k must be a odd integer. However, there can still be ties when $\mathrm{k}$ is an odd integer when performing multiclass classification. After we convert each image to a vector of fixed-length with real numbers, we used the most common distance function for KNN which is Euclidean distance:

$$
d(x, y)=\|x-y\|=\sqrt{(x-y) \cdot(x-y)} \quad=\left(\sum_{i=1}^{m}\left(\left(x_{i}-y_{i}\right)^{2}\right)\right)^{\frac{1}{2}}
$$

Where $x$ and $y$ are histograms in $X=$ ? 0 . Figure 4 below shows visualizes the process of KNN classification.

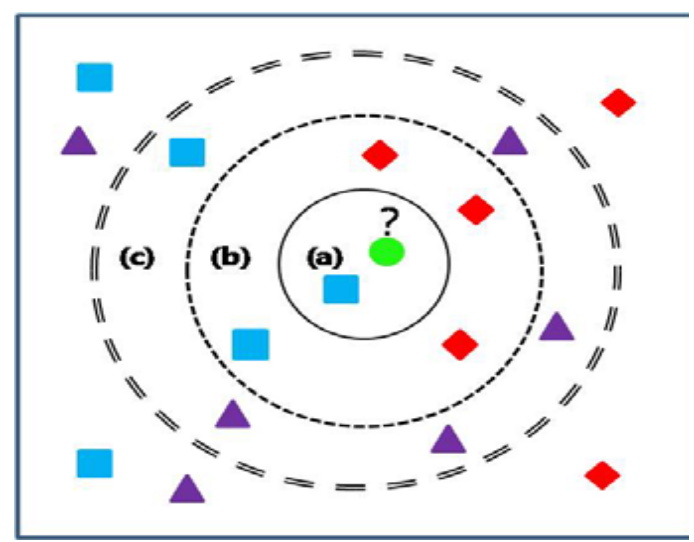

Figure 4. Module $3 \mathrm{~K}-\mathrm{NN}$

At the query point of the circle depending on the $k$ value of 1,5 , or 10 , the query point can be a rectangle at (a), a diamond at (b), and a triangle at (c).

A main advantage of the KNN algorithm is that it performs well with multi-modal2 classes because the basis of its decision is based on a small neighbourhood of similar objects. Therefore, even though the target class is multi-modal, the algorithm can still lead to good accuracy. However a major disadvantage of the KNN algorithm is that it uses all the features equally in computing for similarities. This can lead to classification errors, especially when there is only a small subset of features that are useful for classification.

Module 6: Fuzzy classifier-The sequence of operations in a fuzzy system can be described by three phases, namely, fuzzification, inference, and defuzzification [16]. Design steps for the fuzzy guidance law will be introduced in the following sections.

\section{(i) Fuzzification}

The input and output variables of a fuzzy system are the linguistic variables because they take linguistic values. The input linguistic variables of fuzzy logic-based classification are mean, variance and entropy and the output variable is class The universe of discourse of the 
linguistic variable mean is supposed to be [ 150, 250] deg, variance is [ 0,10$]$ deg,entropy is $[2,8] \mathrm{deg}$ and class is[ 0,3]deg. The fuzzification of the input variables for the classification is denoted in the figure 5 respectively, thus after denoting fuzzification the membership function of each input variable is obtained within their respective range

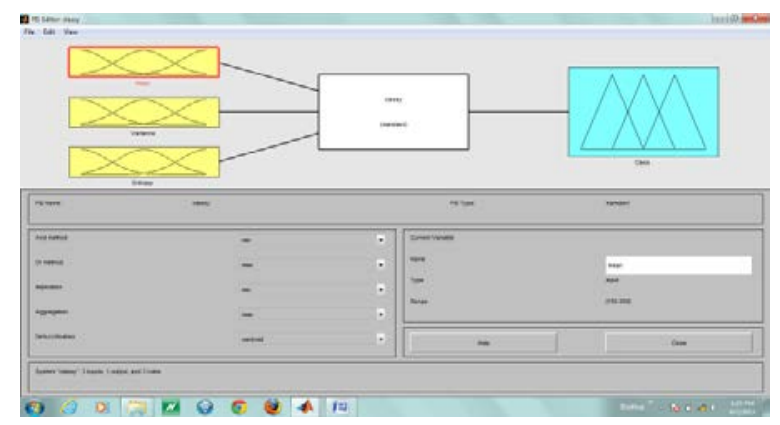

Figure 5. Fuzzification Input Variables

\section{Membership Functions:}

The following membership function for fuzzy mean, variance and entropy are given in below in Figure 6. The Figure 6(a) denotes membership function for mean. The Figure 6(b) denotes membership function for variance. The Figure 6(c) denotes membership function for entropy. And finally 6(d) denotes the overall function for class with respective ulcers

\section{Fuzzy for Mean}

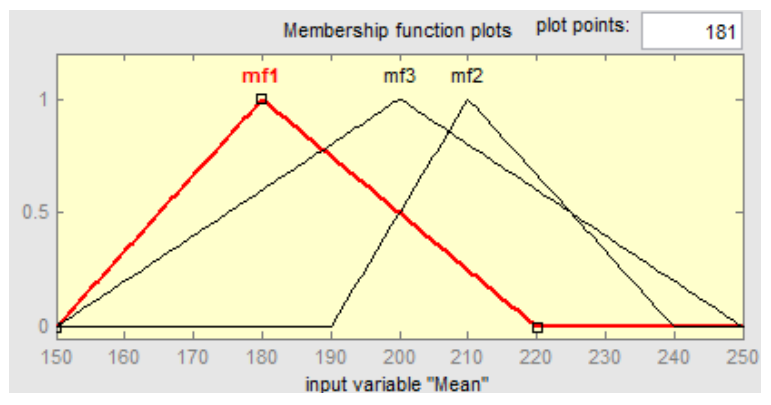

Figure 6(a). Membership Function for Mean

\section{Fuzzy for Variance}

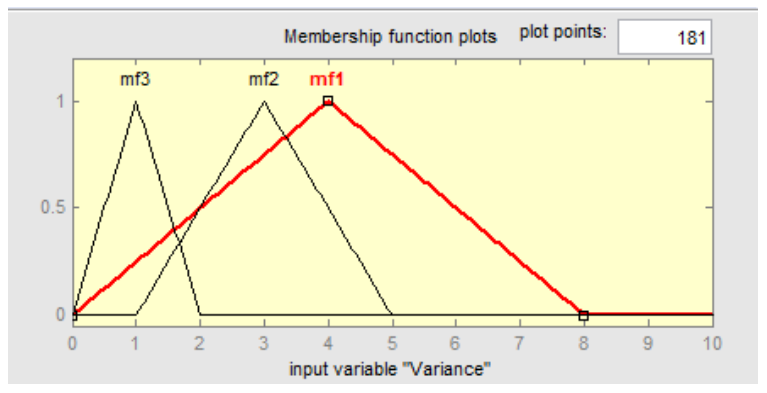

Figure 6(b). Membership Function for Variance 


\section{Fuzzy for Entropy}

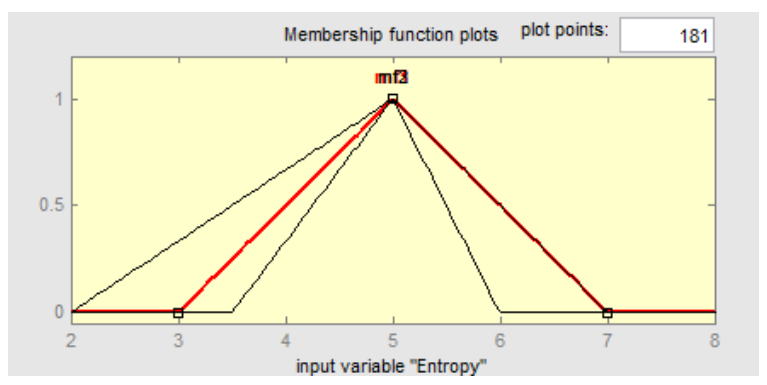

Figure 6(c). Membership Function for Entropy

Fuzzy for Class

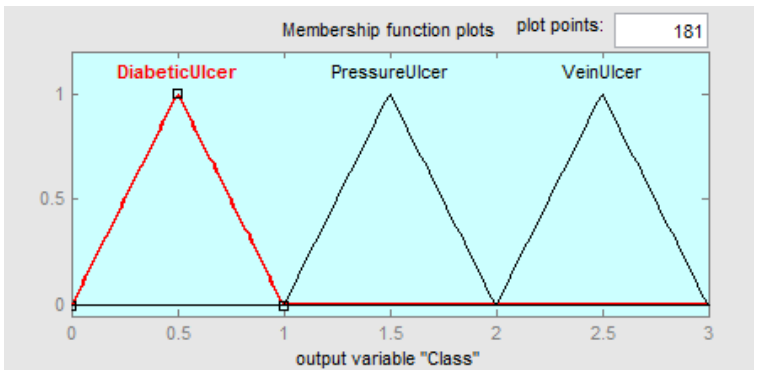

Figure 6(d). Membership Functions for Class

Rule Base: The rule base contains a collection of rules and forms an integral part of the total knowledge for the classification and the following rules are

\section{If (Mean is $\mathrm{mf} 1)$ and (Variance is $\mathrm{mf} 1)$ and (Entropy is $\mathrm{mf} 1)$ then (Class is Diabetic} Ulcer)(1)

\section{If (Mean is $\mathrm{mf} 2$ ) and (Variance is $\mathrm{mf} 2$ ) and (Entropy is $\mathrm{mf} 2)$ then (Class is Pressure Ulcer)(1)}

\section{If (Mean is $\mathrm{mf3}$ ) and (Variance is $\mathrm{mf} 3$ ) and (Entropy is $\mathrm{mf} 3$ ) then (class is Venous Ulcer)(1)}

\section{(ii) Rule Evaluation (Inference)}

We use the max-min (Mamdani-Assilian type) inference16 to generate the best possible conclusions. In this inference mechanism, the min and max operations are used for the AND and OR operations respectively. This type of inference is easy to compute and effective, therefore it is appropriate for real-time control applications. The fuzzified inputs are according to each rule individually. The clipped membership functions of the individual rule are then merged to produce one final fuzzy set. The max operation is used to merge overlapping regions.

\section{(iii) Defuzzification:}

The outputs of the linguistic rules are fuzzy, but the guidance command should be crisp. Therefore, the outputs of the necessary linguistic rules must be defuzzified before feeding into 
the plant. The crisp control action is calculated here using the c.g.center-of-area (COA) Defuzzification procedure. The criterion provides defuzzified output with better continuity. This criterion will be more appropriate than other defuzzification methods.

\section{Fuzzy Surface Graph: Result}

The results of the following is been carried out in Matlab and thus provides the obtained wound type according to the two classifiers used i.e., KNN and Fuzzy classifiers and based on the linguistic input variables and obtained values of the membership functions of mean, variance and entropy. We obtained the results of the class of wound whether the image is diabetic, pressure or venous ulcers, and approximate percentage of healing can b estimated. The surface graph of the results we obtain from the is shown in below Figure 7

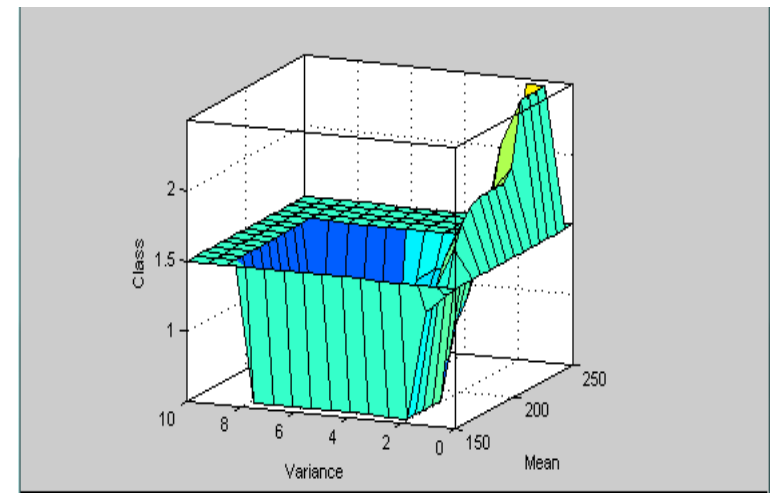

Figure 7. Surface Graph Result

\section{Wound Images Types:}

Wound 1:
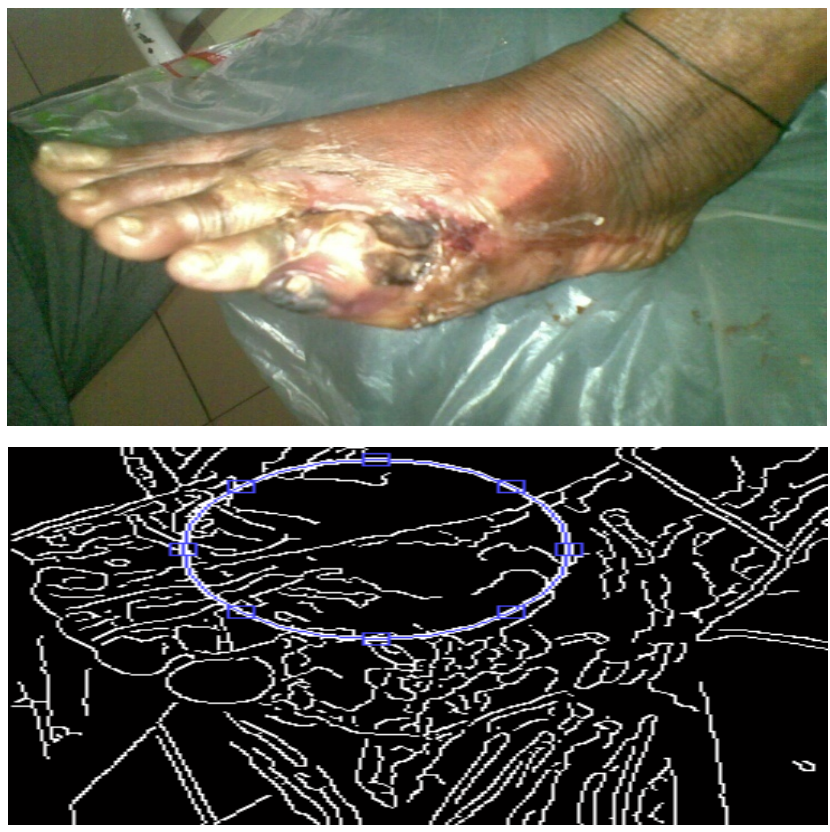
International Journal of Signal Processing, Image Processing and Pattern Recognition Vol.6, No.5 (2013)

\section{Wound 2:}
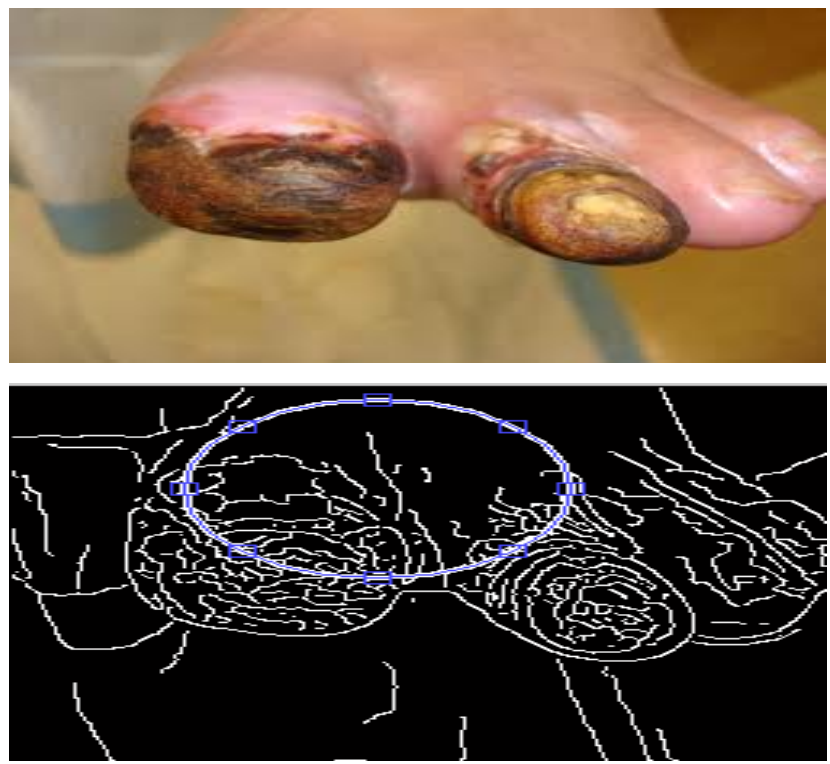

Wound 3:
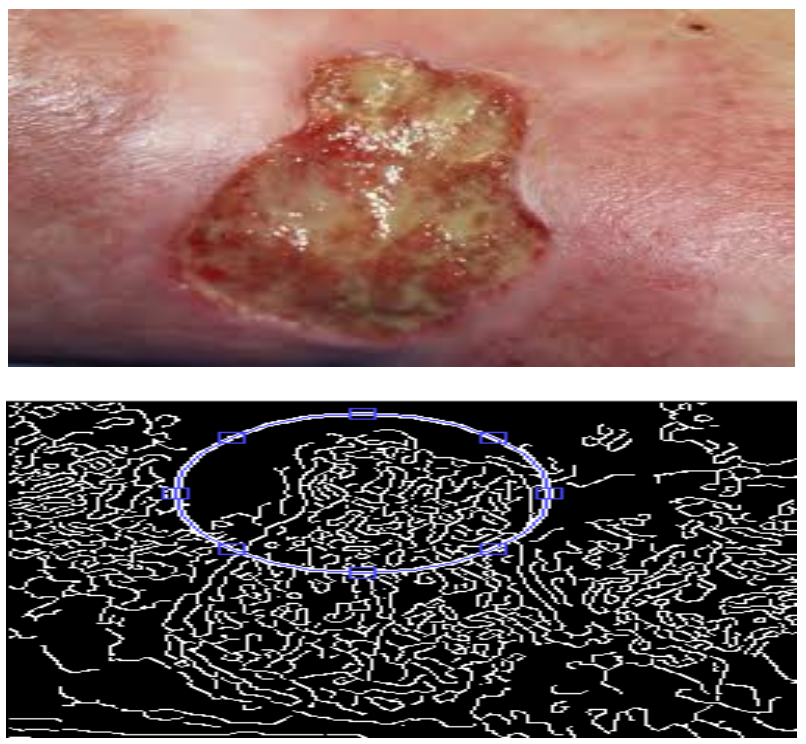

Wound 4:

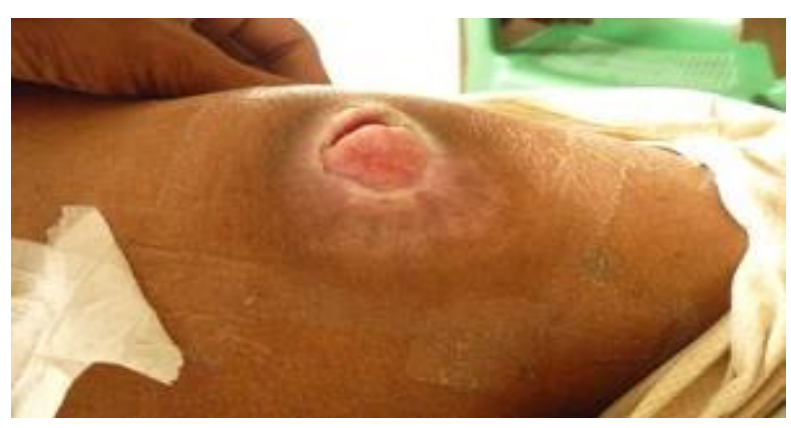




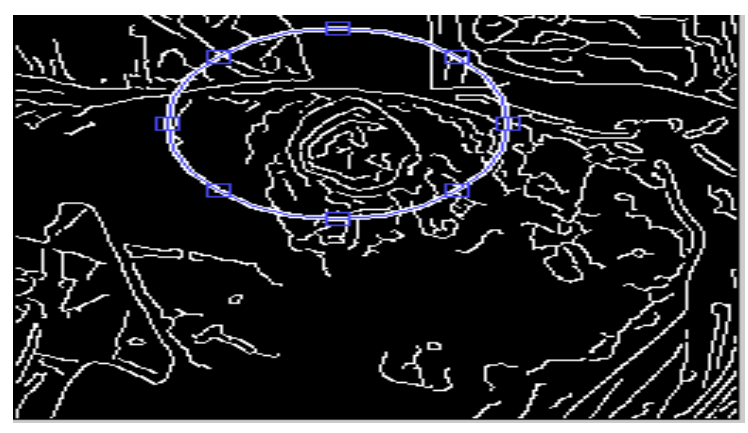

Wound 5:
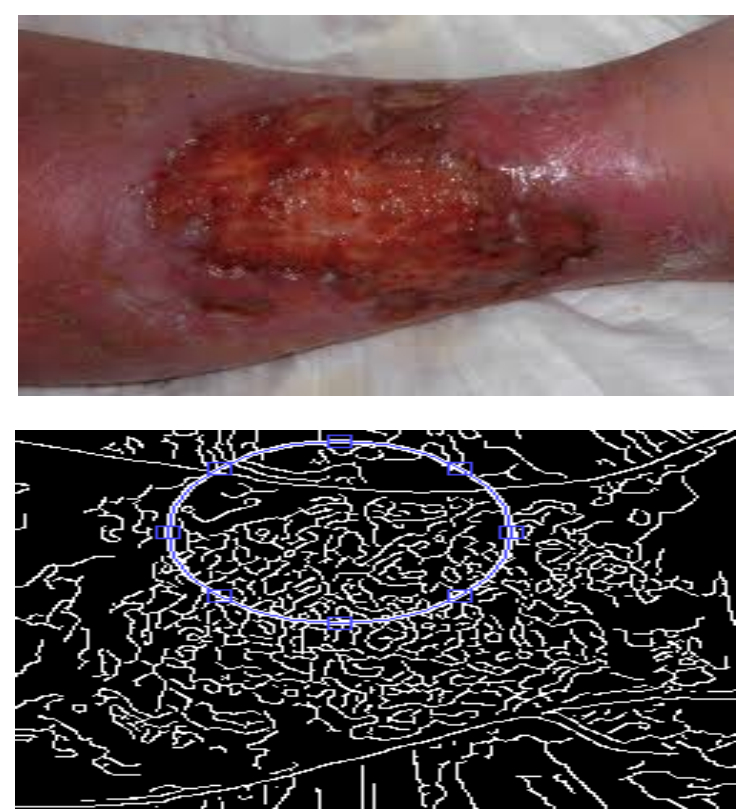

Figure 8. Differents Wound Types and Segmentation

\section{Experimental Results}

\begin{tabular}{|l|l|l|l|}
\hline $\begin{array}{l}\text { Image } \\
\text { name }\end{array}$ & $\begin{array}{l}\text { Fuzzy } \\
\text { Decision }\end{array}$ & $\begin{array}{l}\text { KNN } \\
\text { Decision }\end{array}$ & $\begin{array}{l}\text { \% of } \\
\text { wound } \\
\text { to be } \\
\text { Healed }\end{array}$ \\
\hline Wound 1 & Diabetic & Diabetic & 55.54 \\
\hline Wound 2 & Diabetic & Diabetic & $\mathbf{6 7 . 3 2}$ \\
\hline Wound 3 & Pressure & Pressure & $\mathbf{6 5 . 9 6}$ \\
\hline Wound 4 & Venous & Pressure & $\mathbf{6 9 . 2 6}$ \\
\hline Wound 5 & Venous & Venous & $\mathbf{5 1 . 4 0}$ \\
\hline
\end{tabular}

\section{Conclusion}

In this paper, using the wound assessment for chronic wounds (WAC) and also using certain classifiers i.e., K-NN and Fuzzy are implemented. From the observation of the results, we come to a conclusion that $\mathrm{KNN}$ is most suitable for all types of wounds at the same time 
Fuzzy is suitable for Diabetic and Venous ulcers only since their mean values denotes of higher range which depicts tat wounds may take more time to heal and for other wounds respectively. In our future work we can work mainly in improvising the exact accuracy of the wound type and the healing period, and also use 3 dimensional and also, we can work using some advance machine learning algorithm.

\section{References}

[1] J. J. Dale, “The aetiology of leg ulceration”, Leg Ulcers: nursing management: a research based guide. Cullum, N., Roe, B. Ed. Scutari, London, (1995).

[2] M. Herbin, F. X. Bon, A. Venot, F. Jeanlouis, M. L. Dubertret and D. Strauch, "Assessment of healing kinetics through true colour image processing”, IEEE Transactions on Medical Imaging, vol. 12, no. 1, (1993), pp. 39-43.

[3] J. Mekkes and W. Westerhof, "Image processing in the study of wound healing”, Clinics in Dermatology, vol. 13, no. 4, (1995), pp. 401-407.

[4] B. F. Jones and P. Plassman, “An instrument to measure the dimensions of skin wounds”, IEEE Transactions on Biomedical Engineering, vol. 42, no. 5, (1995), pp. 464-470.

[5] T. A. Krouskop, R. Baker and M. S. Wilson, "A noncontact wound measurement system", Journal of Rehabilitation Research and Development, vol. 39, no. 3, (2002), pp. 337-346.

[6] W. Paul Berriss and S. John Sangwine, “Automatic Quantitative Analysis of Healing Skin Wounds using Colour Digital Image Processing”, World Wide Wounds, Edition, vol. 1, no. 1, (1997).

[7] D. Kane, "Chronic wound healing and chronic wound management”, Krasner D, Rodeheaver GT, Sibbald RG. (eds): Chronic Wound Care: A Clinical Source Book for Healthcare Professionals, Third Edition. Wayne, PA, Health Management Publications, (2001), pp. 7-17.

[8] P. Plassmann, K. G. Harding and J. M. Melhuish, "Methods of Measuring Wound size - A Comparative Study”, WOUNDS, vol. 6, no. 2, (1994), pp. 54-61.

[9] D. H. Keast and C. Keith Bowering, "MEASURE: A proposed assessment framework for developing best practice recommendations for wound assessment”, Wound Repair and Regeneration, vol. 12, no. 1, (2004), pp. 1.

[10] M. E. Roberts and E. Claridge, “An artificially evolved vision system for segmenting skin lesion images”, MICCAI, vol. 1, (2003), pp. 655-662.

[11] T. Gilman, "Wound outcomes: the utility of surface measures”, Lower Extremity wounds, vol. 3, no. 3, (2004), pp. 125-132.

[12] J. Mairal, M. Elad and G. Sapiro, “Sparse Representation for Color Image Restoration”, IEEE Transactions On Image Processing, vol. 17, no. 1, (2008) January.

[13] S. Treuillet, B. Albouy and Y. Lucas, "Three-Dimensional Assessment of Skin Wounds Using a Standard Digital Camera”, IEEE Transactions On Medical Imaging, vol. 28, no. 5, (2009) May.

[14] H. Wannous, Y. Lucas and S. Treuillet, "Enhanced Assessment of the Wound- Healing Processby Accurate Multiview Tissue Classification”, IEEE Transactions On Medical Imaging, vol. 30, no. 2, (2011) February.

[15] H. Wannous, Y. Lucas and S. Treuillet, "Efficient SVM classifier based on colour and texture region features for wound tissue images”, Medical Imaging 2008: Computer-Aided Diagnosis, Proc. of SPIE, vol. 6915, 69152T, (2008).

[16] Feng Chia University, Taichung 40724, Taiwan, Republic of China "Design of Fuzzy Logic Guidance Law against High-Speed Target”, vol. 23, no. 1, (2000) January-February.

\section{Authors}

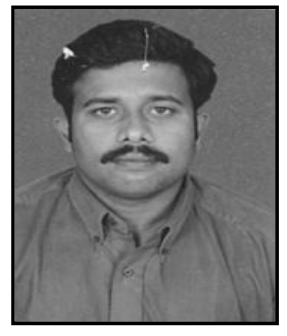

K. Sundeep Kumar received the M.Tech (IT) from Punjabi University in 2003, ME (CSE) from Anna University in 2009 and pursuing Ph. D (CSE) from JNTUA. He is with the department of Computer Science \& Engineering and as an Associate Professor, CMR Institute of Technology, Bangalore. He presented more than 15 papers in International and national Conferences. His research interests include Image Processing, OOMD, Software Engineering and Data Warehousing. He is a life member in ISTE. 


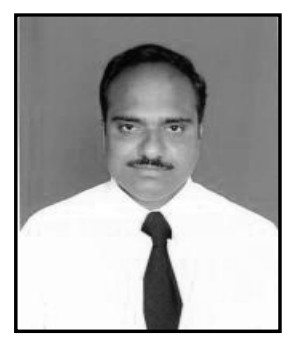

Dr. B. Eswara Reddy Graduated in B.Tech. (CSE) from Sri Krishna Devaraya University in 1995. He received Masters Degree in M.Tech.(Software Engineering), from JNT University, Hyderabad, in 1999. He received Ph.D in Computer Science \& Engineering from JNT University, Hyderabad, in 2008. He served as Assistant Professor from 1996 to 2006. He is working as Professor in CSE Dept., at JNTUACE, Anantapur. He has more than 30 Publications in various International Journals and Conferences. He is one of the author's of the textbook titled Programming with Java published by Pearson/Sanguine Publishers. His research interests include Pattern Recognition\& Image Analysis, Data Warehousing \& Mining and Software Engineering. He is a life member of ISTE, IE, ISCA and member of CSI and IEEE. 
International Journal of Signal Processing, Image Processing and Pattern Recognition Vol.6, No.5 (2013) 